\section{Comparison of the potential acuity meter and the visometer in cataract patients}

O Reid' ${ }^{1}$ DAL Maberley ${ }^{2}$ and H Hollands ${ }^{1}$

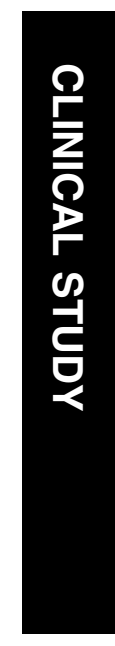

Keywords: cataract; cataract surgery; potential vision; diagnostic tests; surgical outcomes; testing

\section{Introduction}

An accurate assessment of macular function in the presence of cataract is typically performed to determine if surgery will be of benefit.

Preoperative macular assessments include both structural and functional testing. Structural evaluation primarily is performed by slit-lamp biomicroscopy or binocular indirect ophthalmoscopy. Two technologies commonly used for preoperative functional macular assessments include the Snellen chart projector (Potential Acuity Meter (PAM)) and the white-light interferometer (Visometer).

The Guyton-Minkowski PAM works by projecting a miniature Snellen chart through a small window, approximately $0.1 \mathrm{~mm}$ in diameter, in the lens opacity. The image traverses the lens without diffraction from the edges of the aperture or loss of illumination, allowing determination of retinal acuity. ${ }^{1}$

The first quantitative assessment of macular function made by projecting interference fringes onto the retina was described by LeGrand in 1935. ${ }^{2}$ Lotmar documented Moiré fringe testing in 1972 leading to the development of the Visometer, a tool which is able to measure retinal acuity using white light, interferometry in the presence of lenticular, corneal, or vitreous opacities. $^{3,4}$

Although validation studies have been performed for the PAM, ${ }^{5-7}$ no large-scale study has evaluated these two commonly used instruments in a patient population undergoing cataract surgery. The purpose of this study is to analyse the capabilities of both PAM and Visometer in predicting postoperative
${ }^{1}$ Faculty of Medicine, University of British Columbia, Vancouver, Canada

${ }^{2}$ Department of Ophthalmology, University of British Columbia, Vancouver, Canada

Correspondence: DAL Maberley,

Department of Ophthalmology, University of British Columbia, Section C, 2550 Willow Street, Vancouver, BC, Canada V5Z 3N9

Tel: + 604875 4599;

Fax: + 6048754699 .

E-mail: crtg_vancouver@ hotmail.com

Received: 21 January 2005 Accepted in revised form: Published online: 4 November 2005

This research was presented at the University of British Ophthalmology Annual Research Day, May 2002

None of the authors have any financial or proprietary interest in any of the products described in the study 21 September 2005 Columbia Department of 
best-corrected visual acuity (BCVA) and interpret their clinical usefulness in a consecutive series of patients with no known underlying ocular pathology undergoing cataract surgery.

\section{Methods}

From the offices of nine cataract surgeons, a consecutive series of cataract patients who underwent preoperative functional testing at the University of British Columbia/ Vancouver General Hospital Eye Care Centre during a 6-month period in 1999 were evaluated. Ethics approval was received from the University of British Columbia's Clinical Research Ethics Board. Patients with known vision loss from pre-existing macular or optic nerve disease were excluded.

Procedure dates, gender, date of birth, and preoperative visual acuity data, as well as preoperative PAM and Visometer measurements, were collected for each patient. All subjects underwent phacoemulsification with intraocular lens implantation. The nine ophthalmic surgeons performed cataract surgery by a small incision phacoemulsification technique and measured the preand postoperative BCVA. The timing of the final BCVA assessment was left to the discretion of the attending physician.

Preoperative testing was performed by two ophthalmic technicians following identical protocols. The PAM assessment was performed before the Visometer. Both instruments were used in a darkened room, following pupil dilation with Phenylephrine Hydrochloride 5\% and Tropicamide 0.8\%. For the Lotmar Visometer, the power source of the attached Haag-Streit $\mathrm{BM}^{\mathrm{TM}}$ slit-lamp was set at $5.0 \mathrm{~V}$, and the following interferometer settings were chosen: white filter, stop set at $0.5 \mathrm{~mm}$ diameter, acuity scale on 0.05 , and visual field at $3.5^{\circ}$. The acuity scale was advanced by increments of 0.1 from 0.1 to 1.0 or until the patient could no longer identify the orientation of the fringe pattern. ( 0.1 and 1.0 on the Visometer decimal system correspond to 20/200 and 20/20, respectively). For PAM testing, the HaagStreit power input was set at $5.0 \mathrm{~V}$ and subjects were aligned until the projection beam was visible to them. Subjects were tested progressing from largest to smallest acuity lines. A single incorrect response per line was accepted.

Data were entered and analysed using Statistical Package for Social Sciences (SPSS) 10.0. ${ }^{8}$ Best corrected Snellen visual acuity was reported and used for the primary analyses comparing pre- and postoperative visions. Mean postoperative acuities were analysed using the mean values to the Log of the Minimum Angle of Resolution (LogMAR). ${ }^{9}$
Primary analyses included comparisons of (1) preoperative visual acuity estimated using the Visometer and postoperative Snellen BCVA and (2) preoperative visual acuity using the PAM and postoperative Snellen BCVA. Mean values of visual acuities obtained preoperatively by the PAM and the Visometer were compared with the postoperative BCVA using Pearson correlation coefficients. ${ }^{10}$

The accuracy with which the PAM and the Visometer can predict surgical success and failure was compared such that both the PAM and Visometer could be thought of as 'diagnostic tests' being compared to the postoperative BCVA as the 'gold standard'. For this comparison, a successful surgery was defined as a BCVA of 20/40 or better (either predicted or actual), whereas a surgical failure was defined as a BCVA of less than 20/40 (either predicted or actual). Sensitivity of the diagnostic test (either PAM or Visoumeter) was calculated as the proportion of eyes that were predicted to have 'successful surgery' amongst the total number of eyes that were postoperatively found to have undergone 'successful surgery'. Sensitivity, specificity, predictive values, and likelihood ratios were calculated for both PAM and Visometer as diagnostic tests in predicting postoperative surgical success. For sensitivity, specificity, and predictive values, $95 \%$ confidence intervals are reported. ${ }^{10}$ In addition, a multivariate logistic regression analysis was used to predict 'postoperative surgical success' using the PAM and Visometer predictions while controlling for age, gender, and the number of eyes each subject had in the study simultaneously.

\section{Results}

In total, 557 PAM and Visometer tests were performed during the 6-month study period. Tests from nonparticipating physicians $(n=107)$ and those with known macular or optic nerve pathology were excluded $(n=143)$. This left 307 tests for evaluation that represented single eyes of 223 subjects (139 subjects had one study eye included and 84 subjects had two study eyes included). Of the 307 eyes considered as data points for the analysis, four Visometer prediction values were missing and 11 PAM prediction values were missing. Consequently, PAM predictions were compared to postoperative BCVA using 296 eyes, Visometer predictions were compared to postoperative BCVA using 303 eyes, and PAM and Visometer predictions were compared using 292 eyes.

Of the 223 eligible subjects, 136 (61\%) were female and $87(39 \%)$ were male. The mean age of the subjects was 75.5 years $(\mathrm{SD}=10.0$ years) and ranged from 32 to 93 years. The mean follow-up time prior to recording the final postoperative BCVA was 11 weeks (range 4-52). 
The mean true postoperative BCVA was 0.16 LogMAR units $(\mathrm{SD}=0.16)$. The PAM and Visometer estimates of postoperative BCVA were $0.23(\mathrm{SD}=0.35)$ and 0.12 $(\mathrm{SD}=0.29)$ LogMAR, respectively. Preoperative PAM predictions tended to overestimate true postoperative BCVA (mean overestimation $=0.07$ ), whereas Visometer tended to underestimate postoperative BCVA (mean underestimation $=0.04$ ). Table 1 shows the distribution of postoperative acuities compared to PAM and Visometer predictions - measured as the difference in lines of vision between predicted and measured acuities.

There was a rough correlation between postoperative BCVA and PAM predictions (Pearson Correlation, $\rho=0.206, P<0.01)$ and between postoperative BCVA and Visometer predictions (Pearson Correlation, $\rho=0.222$, $P<0.01$ )

The accuracy with which the PAM and the Visometer can predict surgical success and failure was compared and is shown in Table 2. Table 3 shows sensitivity, specificity, predictive values, and likelihood ratios for both the PAM and Visometer as 'diagnostic tests' for

Table 1 Deviation of postoperative acuities from PAM and visometer predictions

\begin{tabular}{lrcrcr}
\hline & Vision & $\begin{array}{c}\text { PAM } \\
\text { number of } \\
\text { subjects }\end{array}$ & $\begin{array}{r}\text { \% of } \\
\text { total }\end{array}$ & $\begin{array}{c}\text { Visometer } \\
\text { number of } \\
\text { subjects }\end{array}$ & $\begin{array}{c}\% \text { of } \\
\text { total }\end{array}$ \\
\hline No. of lines & $>4$ & 20 & 6.76 & 6 & 1.98 \\
Better than & 3 & 14 & 4.73 & 1 & 0.33 \\
Prediction & 2 & 21 & 7.09 & 10 & 3.30 \\
& 1 & 67 & 22.64 & 40 & 13.20 \\
& & & & & \\
No. of lines & -1 & 62 & 20.95 & 94 & 31.02 \\
Worse than & -2 & 23 & 7.77 & 50 & 16.50 \\
Prediction & -3 & 8 & 2.70 & 18 & 5.94 \\
& -4 & 4 & 1.35 & 7 & 2.31 \\
\hline
\end{tabular}

Table 2 Accuracy of the PAM and visometer in predicting surgical success ${ }^{\mathrm{a}}$ and failure

\begin{tabular}{lccc}
\hline & $\begin{array}{c}\text { Postoperative } \\
\text { success }\end{array}$ & $\begin{array}{c}\text { Postoperative } \\
\text { failure }\end{array}$ & Total \\
\hline PAM predictions & & & \\
$\quad$ Predicted success & 232 & 16 & $248(83.8 \%)$ \\
$\begin{array}{l}\text { Predicted failure } \\
\text { Total }\end{array}$ & 42 & 6 & $48(16.2 \%)$ \\
Visometer predictions & $274(92.6 \%)$ & $22(7.4 \%)$ & 296 \\
$\begin{array}{l}\text { Predicted success } \\
\text { Predicted failure }\end{array}$ & 270 & & \\
Total & 11 & 20 & $290(95.7 \%)$ \\
\hline
\end{tabular}

${ }^{\text {aS }}$ urgical success was defined as postoperative BCVA 20/40 or better.
Table 3 Sensitivity and specificity of PAM and visometer in predicting surgical success ${ }^{\mathrm{a}}$ and failure

\begin{tabular}{lcc}
\hline & PAM & Visometer \\
\hline Sensitivity & $84.7 \%(79.7-88.6 \%)$ & $96.1 \%(92.9-97.9 \%)$ \\
$\begin{array}{l}\text { Specificity } \\
\text { Positive predictive } \\
\text { value }\end{array}$ & $93.3 \%(11.6-50.4 \%)$ & $9.1 \%(1.6-30.6 \%)$ \\
$\begin{array}{l}\text { Negative predictive } \\
\text { value }\end{array}$ & $12.5 \%(5.2 \%-96.1 \%)$ & $93.1(89.4-95.6 \%)$ \\
$\begin{array}{l}\text { Likelihood ratio } \\
\text { (success) }\end{array}$ & 1.16 & $15.4 \%(2.7 \%-46.3 \%)$ \\
$\begin{array}{l}\text { Likelihood ratio } \\
\text { (failure) }\end{array}$ & 560 & 1.05 \\
\hline
\end{tabular}

${ }^{\text {aS }}$ urgical success was defined as postoperative BCVA $20 / 40$ or better.

predicting postoperative BCVA ('surgical success' or 'surgical failure'). Over $92 \%$ of surgeries were postoperatively considered 'successful'.

In addition, a multivariate logistic regression analysis was performed in order to predict postoperative success or failure based on the PAM and Visometer predictions of success or failure after controlling for age, gender, and whether the patient had one or two eyes involved in the study. This analysis showed that none of the above variables could significantly predict postoperative success or failure.

\section{Discussion}

Many authors have used case-series data to evaluate the Guyton-Minkowski PAM for predicting postoperative visual acuity after cataract surgery ${ }^{1,5-7}$ or to compare this device to other instruments for predicting postoperative visual acuity after cataract surgery. ${ }^{11-16}$ Two studies have specifically compared the PAM to the Lotmar Visometer. ${ }^{17,18}$ However, these studies employed relatively small sample sizes ( $<55$ each) and a significant proportion of these samples (30-45\%) were made up of subjects with known posterior segment pathology. We also believe the interpretations provided by these studies as to the usefulness of the PAM and Visometer were not complete because neither study evaluated these devices in the setting of the preoperative decision-making process for cataract surgery.

The accuracy of both the PAM and the Visometer in predicting postoperative visual acuity in a large consecutive series of cataract patients with no known vision loss from pre-existing eye disease has been previously unknown. The question is important because the comparative strengths and shortcomings of these two instruments, as they relate to cataract pathology, cannot be easily evaluated in the presence of confounding posterior segment pathology. By evaluating a population 
with no known macular pathology, the effect of cataract alone on the performance of each instrument, individually and in comparison, can be evaluated.

We found a statistically significant correlation between both PAM and Visometer with postoperative BCVA, yet the correlation coefficient was poor and thus clinically (or practically) insignificant. In general, the Visometer slightly underestimated the postoperative acuity, but was closer to the postoperative acuity than the PAM. These trends are similar to previous research on this subject. ${ }^{17,18}$

PAM and Visometer both have high sensitivity and low specificity for predicting surgical success/failure. In this context, sensitivity is defined as the proportion of patients who were predicted to have a successful surgery out of all patients who had a postoperatively defined successful surgery, whereas specificity is the proportion of patients who were predicted to have a failed surgery out of all patients who had a postoperatively defined failed surgery. These measures, however, are not necessarily applicable in a preoperative setting because the true outcome of the surgery is not known preoperatively.

The positive predictive value (PPV) in this situation is the proportion of postoperatively defined successful surgeries out of all patients who were predicted to have a successful surgery with PAM or Visometer. The negative predictive value (NPV) is the proportion of postoperatively defined failed surgeries, which were predicted to fail preoperatively with PAM or Visometer. The PPV and NPV for both the instruments were high and low, respectively. In general, PPV and NPV are more applicable to the preoperative situation. For instance, if a patient has a preoperative test (PAM or Visometer) that predicts surgical success the PPV gives the clinician an estimate of the probability of surgical success. However, PPV and NPV both necessarily depend on the overall chance of surgical success based on the population of interest. ${ }^{19}$

Based on our data, $92.7 \%$ of all surgeries performed were successful. If a patient had a positive prediction from PAM or Visometer, the chance of success (ie PPV) was marginally increased from 92.7 to $93.1 \%$ for the PAM and from 92.7 to $93.5 \%$ for the Visometer. Stated another way, the positive likelihood ratio (PLR), or the odds that a predicted surgical success would be expected in a patient who has a true surgical success (compared to a true surgical failure) is essentially 1.0. Thus, a preoperative prediction of surgical success from either PAM or Visometer is of no clinical value.

Conversely, if the PAM or Visometer preoperatively predicted a negative surgical outcome, the chance of surgical success was still over $84 \%$ and surgical intervention would universally be considered appropriate. Furthermore, if a preoperative prediction of surgical failure led to a decision by an ophthalmologist not to extract a patient's cataract then a patient with a high likelihood of benefit from intervention would not receive surgery.

The reason for this finding is that the PPV of preoperative testing in this setting necessarily rises with the overall proportion of successful cataract surgery (likewise, the NPV necessarily decreases). In our study, the PPV is high and NPV is low mostly because our pre-test likelihood of surgical success is high $(92.7 \%$ of 'all-comers' had successful surgery). We have already shown how preoperative testing with PAM and Visometer are of negligible benefit in the setting of a high pre-test likelihood of surgical success. However, a diagnostic test is most useful if the preoperative probability is close to $50 \%$ (in this situation if there were a $50 \%$ pretest likelihood of surgical success). The likelihood ratios for the PAM and Visometer can be used to predict post-test likelihood of surgical success/failure from the pretest likelihood independent of the overall likelihood of surgical success. In our study, both positive and negative likelihood ratios are close to 1.0, making their predictive utility of little clinical value. Therefore, in patients undergoing cataract surgery that has no identified retinal or macular pathology, the PAM and Visometer have little usefulness and are potentially detrimental in the preoperative setting.

There are limitations to our study design. Firstly, we evaluated a population of patients who had been sent for preoperative functional testing at the discretion of their ophthalmologist. Therefore, there is potential for bias if this population differs in how PAM and Visometer could predict postoperative outcome compared to a random population of patients undergoing cataract surgery (who may or may not have been sent for preoperative functional testing at their ophthalmologists discretion). However, by examining a population of patients who have been chosen to undergo preoperative functional testing we were able to look at the effectiveness - or usefulness in a real world setting - of these assessment tools. A second limitation of this study is that we are assessing patients with no known macular pathology but we did not perform a universal retinal exam on each patient as part of the study design. Thirdly, we do not take visual acuity data from standardized postoperative time points and did not specifically account for early posterior capsular opacification or surgery-related complications.

Our study provides quite different conclusions when compared to previously published reports on this subject. ${ }^{17,18}$ First, we found no overall significant difference between the PAM and Visometer in preoperative predictive ability, but this point is of limited value since neither instrument predicted well. Second, 
we were unable to find evidence of any clinical benefit to using the PAM or Visometer in the setting of preoperative cataract assessment where the retinal evaluation is grossly normal. We recommend that these preoperative instruments not be used for this purpose due to cost and the potential for withholding potentially vision-saving treatment from patients who may benefit. In conclusion, the use of the Potential Acuity Meter or Visometer in preoperative cataract assessment does not provide a clinically useful prediction of postoperative best-corrected visual acuity.

\section{Acknowledgements}

Funding was provided by a UBC Summer Student Research Grant.

\section{References}

1 Minkowski JS, Palese M, Guyton D. Potential acuity meter using a minute aerial pinhole aperture. Ophthalmol 1983; 90(11): 1360-1368

2 LeGrand Y. Sur la mesure de l'acuite oculaire au moyen de franges d'interference. CR Acad Sci 1935; 200: 490.

3 Lotmar W. Use of moiré fringes for testing visual acuity of the retina. Appl Optics 1972; 11: 1266-1268.

4 Lotmar W. Apparatus for the measurement of retinal visual acuity by moiré fringes. Invest Ophthalmol Vis Sci 1980; 19: 393-401.

5 Severin TD, Severin SL. A clinical evaluation of the potential acuity meter in 210 cases. Annals Ophthalmol 1988; 20: 373-375.

6 Gus PI, Kwitko I, Roehe D, Kwitko S. Potential acuity meter in cataract patients. J Cataract Refract Surg 2000; 26(8): 1238-1241.
7 Deverau CJ, Rando A, Wagstaff CM, Story IH. Potential acuity meter results in cataract patients. Clin Exp Ophthalmol 2000; 28(6): 414-418.

8 SPSS For Windows, 9th ed. SPSS Inc.: Chicago, 1998.

9 Steinberg EP et al. The VF-14, an index of functional impairment in patients with cataract. Arch Ophthalmol 1994; 112: $630-638$.

10 Fleiss J. Statistical Methods for Rates and Proportions. John Wiley and Sons: New York, 1981.

11 Cuzzani OD, Ellant JP, Young PW, Gimbel HV, Rydz M. Potential acuity meter versus scanning laser ophthalmoscope to predict visual acuity in cataract patients. J Cataract Refract Surg 1998; 24: 263-269.

12 Tharp A, Cantor L, Yung C, Shoemaker J. Prospective comparison of the Heine Retinometer with the potential acuity meter for the assessment of potential visual acuity before cataract surgery. Ophthalmic Surgery 1994; 25(9): 576-579.

13 Miller ST, Graney MJ, Elam JT et al. Predictions of outcomes from cataract surgery in elderly persons. Ophthalmol 1988; 95: 1125-1129.

14 Datiles MB, Edwards PA, Kaiser-Kupfer MI et al. A comparative study between the PAM and the laser interferometer in cataracts. Graefe's Archive Clin Experimental Ophthalmol 1987; 225: 457-460.

15 Lasa MSM, Datiles MB, Freidlin V. Potential vision tests in patients with cataracts. Ophthalmol 1995; 102: 1007-1011.

16 Barrett BT, Davison PA, Eustace P. Clinical comparison of three techniques for evaluating visual function behind cataract. Eye 1995; 9: 722-727.

17 Spurny RC, Zaldivar R, Belcher D, Simmons RJ. Instruments for predicting visual acuity. Arch Ophthalmol 1986; 104: 196-200.

18 Le Sage C, Bazalgette C, Arnaud B, Shmitt-Bernard C. Accuracy of the IRAS GT interferometer and potential acuity meter prediction of visual acuity after phacoemulsification. J Cataract Refract Surg 2002; 28: 131-138.

19 Sackett DL, Haynes RB, Guyatt GH, Tugwell P. Clinical Epidemiology: a basic science for clinical medicine. 2nd edn, Little, Brown and Co: Boston, 1991. 